\title{
Relaxation Processes in Many Particle Systems - Recurrence Relations Approach
}

\author{
Anatolii V. Mokshin* \\ Kazan Federal University, Kazan, 420008, Russia
}

\begin{abstract}
The general scheme for the treatment of relaxation processes and temporal autocorrelations of dynamical variables for many particle systems is presented in framework of the recurrence relations approach. The time autocorrelation functions and/or their spectral characteristics, which are measurable experimentally (for example, due to spectroscopy techniques) and accessible from particle dynamics simulations, can be found by means of this approach, the main idea of which is the estimation of the so-called frequency parameters. Model cases with the exact and approximative solutions are given and discussed.
\end{abstract}

\section{INTRODUCTION}

Relaxation processes, which emerge in many particle systems, are characterized by highly nontrivial features even for the cases of the well-known simplified models [1]. So, for example, the ideal gas dynamics at the drive by external fields exhibit the non-Markovian (memory) effects [2] as well as the manifestations of anomalous transport [3], whilst the chain of coupled harmonic oscillators can display the nonlinear dynamics [4] with stochastic resonance peculiarities [5]. At the presence of complicated fields of interactions in many particle systems together with structural disorder and intricate spatiotemporal correlations allows one to recognize these as the complex systems. Thus, dense liquids, structural and spin glasses, foams, emulsions and colloidal gels are the typical examples of the physical complex systems, which combine the complicated dynamics together with the structural inhomogeneity [6].

From theoretical standpoint, the description of many particle dynamics reduces oneself into a unified fashion at the applying the mathematical language of the distributions, the correlation and relaxation functions, as well as the Green functions, that provide a statistical treatment to some extent. Berne and Harp marked the significance of time correlation functions in the consideration of dynamic processes by the phrase [7]: ". . time correlation functions have done for the theory of time-dependent processes what the partitions functions have done for the equilibrium theory. The time-dependent problem has became well defined ...". These enthusiastic words become more clear and accepted, if one takes into account that the correlation functions appear to be directly related with the experimentally measured quantities due to Kubo's linear response theory [8] as well as by means of nonlinear response approach [9], which obtained recently a rapid development. Importantly, the time correlation functions are associated with the concrete relaxation processes and, thereby, provide the information about the proper relaxation time scales [2]. Moreover, these functions can be applied to estimate quantitatively and simply the so-called memory effects in many particle system dynamics [2], the dynamical heterogeneity effects in particle movements [10] and the breakdown of system ergodicity 11.

Historically, the formulation of fluctuation-dissipation theorem [12] and the Zwanzig-Mori's projection operator formalism [1] can be distinguished as the important milestones in development of the theoretical description within time correlation and relaxation functions concept. If the above-mentioned theorem was a forthright indication on the relation between a (system) response function to an external field and the fluctuations of the corresponding degree of freedom, then the projection operator formalism was the first one, which had provided us by a general scheme to define the time correlation functions at the consideration of many particle system dynamics within unified mathematical constrains. The projection operator technique allowed one to establish the exact relations between characteristics of the different relaxation processes, that had formed later a basis for the construction of such theories like the generalized hydrodynamics [13] and the mode-coupling theories [14, 15]. Despite obvious advances of the ZwanzigMori's projection operator formalism in the description of many particle system dynamics, the universal mathematical patterns in these systems became more clear with the appearance of the recurrence relations approach [16, 17]. In particular, this approach gave a clear idea that the (dis)similarity of relaxation time scales and the structural recursive nature - structural hierarchy - can be mathematically taken into account by means of recurrence relations.

The aim of this paper is to show the general scheme how the relaxation processes in many particle systems can be treated within the recurrence relations approach. Section 2 presents the some typical relations between the experimentally measured terms and the correlation functions, which describe relaxation processes in many particle

*Electronic address: anatolii.mokshin@mail.ru 
systems. The formulation of the basic general relations is given in section 3 . The simplified model cases with the exact solutions are presented in sections 4 and 5, whereas the description of the density fluctuations in simple liquids is considered in section 6. And, finally, we conclude in Section 7.

\section{CORRELATION FUNCTIONS VS. OBSERVABLE TERMS}

Let us consider the system of $N$ interacted particles, which evolves at the temperature $T$ within the volume $V$. The full dynamics of the system is characterized by a set of the variables $A_{n}(t)$ like, for instance, local density, particle displacement, particle velocity, dipole moment etc. Nevertheless, a concrete problem under study enforces, as a rule, to restrict oneself by a some variable associated with phenomena. The choice of the dynamical variable can be caused by the experimentally measurable response function, which is related with the corresponding relaxation (or correlation) function of this dynamical variable.

Scattering techniques. - The inelastic neutron scattering and the inelastic X-ray scattering techniques allow one to measure the dynamic structure factor $S(k, \omega)$ and the incoherent scattering function $S_{s}(k, \omega)$, where $k$ is the wave number and $\omega$ is the frequency. These terms are related with the autocorrelation functions of the spatial Fourier transforms for the local density fluctuations $\rho(\mathbf{r}, t)=(1 / \sqrt{N}) \sum_{i} \delta\left[\mathbf{r}-\mathbf{r}_{i}(t)\right]$ and for the tagged (single) particle displacement $\rho_{s}(\mathbf{r}, t)=\delta\left[\mathbf{r}-\mathbf{r}_{i}(t)\right]$, correspondingly, [13]

$$
\begin{aligned}
& S(k, \omega)=\frac{S(k)}{2 \pi N} \int_{-\infty}^{\infty} d t e^{-i \omega t} \sum_{i, j} \frac{\left\langle e^{-i \mathbf{k} \cdot \mathbf{r}_{i}(0)} e^{i \mathbf{k} \cdot \mathbf{r}_{j}(t)}\right\rangle}{\left\langle e^{-i \mathbf{k} \cdot\left[\mathbf{r}_{i}(0)-\mathbf{r}_{j}(0)\right]}\right\rangle} \\
& S_{s}(k, \omega)=\frac{1}{2 \pi N} \int_{-\infty}^{\infty} d t e^{-i \omega t} \sum_{i}\left\langle e^{-i \mathbf{k} \cdot \mathbf{r}_{i}(0)} e^{i \mathbf{k} \cdot \mathbf{r}_{i}(t)}\right\rangle,
\end{aligned}
$$

where $\mathbf{k}$ is the wave vector, $S(k)=(1 / N) \sum_{i, j}\left\langle e^{-i \mathbf{k} \cdot\left[\mathbf{r}_{i}(0)-\mathbf{r}_{j}(0)\right]}\right\rangle=\int_{-\infty}^{\infty} d \omega S(k, \omega)$ is the static structure factor, $k=|\mathbf{k}|$ and $\rho(\mathbf{k}, t)=(1 / \sqrt{N}) \sum_{i} e^{i \mathbf{k} \cdot \mathbf{r}_{i}(t)}$ is the space Fourier transform of $\rho(\mathbf{r}, t)$. Thus, the dynamic structure factor $S(k, \omega)$ estimates the collective dynamics with frequencies $\omega$ over spatial scales $\sim 2 \pi / k$ and is related with the density autocorrelation function (or the so-called intermediate scattering function)

$$
\phi_{c o h}(k, t)=\frac{1}{N} \sum_{i, j} \frac{\left\langle e^{-i \mathbf{k} \cdot \mathbf{r}_{i}(0)} e^{i \mathbf{k} \cdot \mathbf{r}_{j}(t)}\right\rangle}{\left\langle e^{-i \mathbf{k} \cdot\left[\mathbf{r}_{i}(0)-\mathbf{r}_{j}(0)\right]}\right\rangle},
$$

whereas the incoherent scattering function $S_{s}(k, \omega)$ performs the same for single particle dynamics and is associated with the particle displacement autocorrelation function (the self-intermediate scattering function)

$$
\phi_{\text {self }}(k, t)=\frac{1}{N} \sum_{i}\left\langle e^{-i \mathbf{k} \cdot \mathbf{r}_{i}(0)} e^{i \mathbf{k} \cdot \mathbf{r}_{i}(t)}\right\rangle .
$$

Dielectric spectroscopy. - The complex dielectric permittivity $\varepsilon^{*}(\omega)$ is measurable due to dielectric spectroscopy experiments. This term is related with the macroscopic dipole correlation function

$$
\phi_{d}(t)=\frac{\langle\mathbf{M}(0) \mathbf{M}(t)\rangle}{\left\langle\mathbf{M}(0)^{2}\right\rangle}
$$

by the relation [18]

$$
1-\frac{\varepsilon^{*}(\omega)-\varepsilon_{\infty}}{\varepsilon_{s}-\varepsilon_{\infty}}=\Im\left[s \int_{0}^{t} d t e^{-s t} \phi(t)\right], s=i \omega
$$

where $\mathbf{M}(t)$ is the macroscopic fluctuating dipole moment of the sample volume unit, which is equal to the vector sum of all the molecular dipoles, $\varepsilon_{s}$ and $\varepsilon_{\infty}$ are the low- and high-frequency limits of the dielectric permittivity, respectively, and $\Im[. .$.$] means imaginary part of [...].$

Transport coefficients. - A feature of resonance techniques is that they can measure the transport properties. So, for example, the nuclear magnetic resonance provides the information about the self-diffusion coefficient $D_{s}$ and viscosity $\eta$ of study system, which are (as well as other transport coefficients) are related with the autocorrelation functions from 
the corresponding current dynamical variables through the Green-Kubo relations [13]. So, for self-diffusion coefficient one has

$$
D=\frac{k_{B} T}{m} \int_{0}^{\infty} d t \frac{\langle\mathbf{v}(0) \mathbf{v}(t)\rangle}{\langle\mathbf{v}(0) \mathbf{v}(0)\rangle}
$$

where

$$
\phi_{v e l}=\frac{\langle\mathbf{v}(0) \mathbf{v}(t)\rangle}{\langle\mathbf{v}(0) \mathbf{v}(0)\rangle}
$$

is the single particle velocity autocorrelation function. Equation, similar to Eq. (77), can be written for rotational diffusion coefficient in term of the dynamical variable - angular velocity $\Omega_{\alpha}$, that accounts the molecular reorientations measurable in the depolarization of fluorescence studies.

Further, the shear viscosity is

$$
\eta=\frac{1}{k_{B} T V} \int_{0}^{\infty} d t\left\langle P_{x y}(0) P_{x y}(t)\right\rangle
$$

where

$$
\phi_{\eta}(t)=\left\langle P_{x y}(0) P_{x y}(t)\right\rangle
$$

is the autocorrelation function of the components of the pressure tensor, which are given by virial formula

$$
P_{\alpha \beta}=\sum_{i=1}^{N}\left(m v_{i \alpha} v_{i \beta}+\frac{1}{2} \sum_{i \neq j}^{N} F_{i j \alpha} r_{i j \beta}\right), \alpha, \beta=x, y, z,
$$

and $F_{i j \alpha}$ denotes the $\alpha$-component of the force between particles $i$ and $j$ that are at distance $r_{i j}$ from one another.

The thermal conductivity $\lambda$ can be expressed as

$$
\lambda=\frac{1}{k_{B} T^{2} V} \int_{0}^{\infty} d t\left\langle J_{0}^{e z}(0) J_{0}^{e z}(t)\right\rangle
$$

where

$$
\phi_{\lambda}=\left\langle J_{0}^{e z}(0) J_{0}^{e z}(t)\right\rangle
$$

is the heat current autocorrelation function with the dynamical variable

$$
J_{0}^{e z}=\sum_{i=1}^{N} v_{i z}\left(\frac{m\left|\mathbf{v}_{i}\right|^{2}}{2}+\frac{1}{2} \sum_{i \neq j}^{N} U\left(r_{i j}\right)\right)-\frac{1}{2} \sum_{i=1}^{N} \sum_{i \neq j}^{N} \mathbf{v}_{i} \mathbf{r}_{i j} \frac{\partial U\left(r_{i j}\right)}{\partial z_{i j}}
$$

$U\left(r_{i j}\right)$ is the potential of particle interaction.

The examples given above show clearly that a variety of quantities experimentally observed can be treated in terms of the time correlation (or relaxation) functions. Relation of other quantities experimentally measured with the corresponding correlation functions can be found, for example, in comprehensive review [7]. Thus, the problem of the explanation of the experimental results in some (not all, but many) cases can be reduced to the problem of the finding either the proper relaxation function or, at least, the asymptotic behavior of this function.

\section{THEORETICAL BACKGROUND}

Let us assume that we consider the dynamical variable $A(t)$, the time evolution of which is defined by the Heisenberg equation

$$
\frac{d A(t)}{d t}=\mathrm{i}[H, A(t)]=\mathrm{i} \hat{\mathcal{L}} A(t),\left.\quad A(t)\right|_{t=0}=A
$$


where $H$ is the Hamiltonian of the system, $\hat{\mathcal{L}}$ is the Liouville operator, which is taken to be Hermitian and [ . . . ] is the Poisson bracket. The formal solution of equation (15) can be written as

$$
A(t)=\mathrm{e}^{\mathrm{i} \hat{\mathcal{L}} t} A .
$$

On the other hand, the Hamiltonian $H$ defines the averaging operation $\langle A\rangle$ through the density of phase space $\rho \propto \exp [-\beta(H-\mu N)]$, where $\mu$ is the chemical potential, $\beta=\left(k_{B} T\right)^{-1}$ and $k_{B}$ is the Boltzmann constant. Then, in classical limit one has a simple correspondence between the scalar product of a pair of dynamical variables $A$ and $B$ of the Liouville space and the corresponding correlation function [13]

$$
(A, B) \equiv\left\langle A B^{*}\right\rangle .
$$

The symbol $*$ marks the complex conjugation. In fact, equation (17) provides the identity between the relaxation function $(A(t), B)$ and the time correlation function $\left\langle A(t) B^{*}\right\rangle$, i.e. $(A(t), B) \equiv\left\langle A(t) B^{*}\right\rangle$. Further, we will utilize the time autocorrelation functions (TACF) in the dimensionless form

$$
\phi(t)=\frac{\left\langle A(0)^{*} A(t)\right\rangle}{\left\langle|A(0)|^{2}\right\rangle}
$$

that ensures the fulfillment of the next conditions

$$
\left.\phi(t)\right|_{t=0}=1, \quad 1 \geq \phi(t) \geq 0,\left.\quad \frac{d \phi(t)}{d t}\right|_{t=0}=0 .
$$

Such a representation of the TACF allows one to focus on the time dependence of $\phi(t)$ directly, and to do the comparison of autocorrelations for different dynamical variables.

Applying the Gram-Schmidt orthogonalization procedure at the initial condition $A_{0} \equiv A$, we generate the set of dynamical variables:

$$
\begin{gathered}
\mathbf{A}=\left\{A_{0}, A_{1}, A_{2}, \ldots, A_{\nu}, \ldots\right\}, \\
\left(A_{\nu}, A_{\mu}\right)=\left(A_{\nu}, A_{\nu}\right) \delta_{\nu, \mu}, \\
\nu, \mu=0,1,2,3, \ldots,
\end{gathered}
$$

which are related by the recurrence relation

$$
\begin{gathered}
A_{\nu+1}=\mathrm{i} \hat{\mathcal{L}} A_{\nu}+\Delta_{\nu} A_{\nu-1}, \\
\Delta_{\nu}=\frac{\left(A_{\nu}, A_{\nu}\right)}{\left(A_{\nu-1}, A_{\nu-1}\right)}, \\
A_{-1}=0, \quad \Delta_{0}=1,
\end{gathered}
$$

where $\Delta_{\nu}$ are the frequency parameters with the dimension of the squared frequency, $\delta_{\nu, \mu}$ is the Kronecker delta. From the definition (21b) one can see that the physical meaning of the parameters $\Delta_{\nu}$ depends on the concrete process and is defined by the corresponding dynamical variables $A_{\nu-1}$ and $A_{\nu}$. Equation (21a) is also known from the recurrence relations approach as the first recurrence relation [17, 19, 20]. Usually, it is convenient (but not necessarily) to perform the construction of the set $\mathbf{A}$ on the basis of the dynamical variable $A_{0}$, which is associated with the processes experimentally studied.

Both the projection operators technique [1] as well as the recurrence relations approach [19, 20] yield the chain of the integro-differential equations for the dynamical variables $\mathbf{A}$ of the form

$$
\begin{aligned}
\frac{d}{d t} A_{\nu}(t)= & -\Delta_{\nu+1} \int_{0}^{t} A_{\nu}(t-\tau) \frac{\left\langle A_{\nu+1}(0)^{*} A_{\nu+1}(\tau)\right\rangle}{\left\langle\left|A_{\nu+1}(0)\right|^{2}\right\rangle} d \tau+A_{\nu+1}(t), \\
& \text { here and hereafter } \nu=0,1,2,3, \ldots,
\end{aligned}
$$

that is the exact consequence of equation (15). ${ }^{1}$ For the TACF's defined as

$$
\phi_{\nu}(t)=\frac{\left\langle A_{\nu}(0)^{*} A_{\nu}(t)\right\rangle}{\left\langle\left|A_{\nu}(0)\right|^{2}\right\rangle}
$$

\footnotetext{
${ }^{1}$ For a case, when the dynamical variable is chosen to be the particle velocity, i.e. $A_{0}=v$, equation (22) represents the known generalized Langevin equation.
} 
the chain (22) takes the following form

$$
\frac{d}{d t} \phi_{\nu}(t)=-\Delta_{\nu+1} \int_{0}^{t} \phi_{\nu}(t-\tau) \phi_{\nu+1}(\tau) d \tau
$$

Then, applying the Laplace transform operator

$$
\hat{\mathcal{L}}[f(\tau)]=\tilde{f}(s)=\int_{0}^{\infty} e^{-s \tau} f(\tau) d \tau
$$

to chain of equations (23), one obtain the recurrence formula

$$
\tilde{\phi}_{\nu}(s)=\frac{1}{s+\Delta_{\nu+1} \tilde{\phi}_{\nu+1}(s)},
$$

which can be transformed to continued fraction representation of frequency spectrum of the TACF:

$$
\tilde{\phi}_{0}(s)=\frac{1}{s+\frac{\Delta_{1}}{s+\frac{\Delta_{2}}{s+\frac{\Delta_{3}}{s+\ddots}}} .}
$$

On the one hand, equation (26) indicates that the form of the spectrum $\tilde{\phi}_{0}(s)$ and, thereby, of the relaxation function $\phi_{0}(t)$, is completely defined by the frequency parameters $\Delta_{\nu}$ as well as by the ratios $\Delta_{\nu+1} / \Delta_{\nu}$. On the other hand, the values of $\Delta_{\nu}$ 's are directly associated with the frequency/time range, for which the fraction solution [equation (26)] will be relevant. Thus, the problem of finding $\phi_{0}(t)\left[\right.$ or $\left.\tilde{\phi}_{0}(s)\right]$ is reduced, mathematically, to the problem of finding a function, which is representable in the form of continued fraction with the some unique set of values of $\Delta_{\nu}$ 's. Although these frequency parameters are physical characteristics of the concrete relaxation processes [see equation (21b)], one can possible to consider some different model situations, which can be realized in some general cases.

\section{MODELS OF THE FINITE SETS OF VARIABLES}

Let us consider the cases with the finite set of dynamical variables ( $\nu$ is finite), that corresponds to the finitedimensional Liouville spaces. Such situations arise at the condition with $A_{\nu}=0$ and $\Delta_{\nu}=0$; and are relevant to nonergodic processes with non-decaying correlation functions expressed by cosine functions.

\section{A. A case of $\nu=2$}

At the condition $\nu=2$ one has $A_{2}=0$ and $\Delta_{2}=0 .{ }^{2}$ Then, continued fraction (26) yields the system of two equations

$$
\left\{\begin{array}{c}
1-s \tilde{\phi}_{0}(s)=\Delta_{1} \tilde{\phi}_{0}(s) \tilde{\phi}_{1}(s), \\
s \tilde{\phi}_{1}(s)=1
\end{array}\right.
$$

with simple solutions in the time domain

$$
\begin{gathered}
\phi_{0}(t)=\cos \left(\sqrt{\Delta_{1}} t\right), \\
\phi_{1}(t)=1 .
\end{gathered}
$$

The relaxation function of the form (28a) reproduces the behavior of undamped harmonic oscillator. This situation is realized, for example, in the case of density fluctuations of homogeneous electron gas at the finite wave numbers $k$ and the temperature $T=0$. Other example, where the case appears, is the dynamics of the chain of classical harmonic oscillators. Here, the TACF of particle velocity, $\phi_{0}(t)=\langle v(0) v(t)\rangle /\left\langle v(0)^{2}\right\rangle$, is described by equation (28a) (see reference [21]).

\footnotetext{
${ }^{2}$ It is clear that the case of $\nu=1$ is trivial. Therefore, this case is not considered here.
} 


\section{B. A case of $\nu=3$}

One has here that $A_{3}=0$ and $\Delta_{3}=0$. Then, continued fraction (26) in this case transforms into the next system of equations:

$$
\left\{\begin{array}{c}
1-s \tilde{\phi}_{0}(s)=\Delta_{1} \tilde{\phi}_{0}(s) \tilde{\phi}_{1}(s), \\
1-s \tilde{\phi}_{1}(s)=\Delta_{2} \tilde{\phi}_{1}(s) \tilde{\phi}_{2}(s), \\
s \tilde{\phi}_{2}(s)=1
\end{array}\right.
$$

which can be resolved by means of the inverse Laplace transform $\hat{\mathcal{L}}^{-1}$ and yields the solutions

$$
\begin{gathered}
\phi_{0}(t)=\frac{1}{\Delta_{1}+\Delta_{2}}\left[\Delta_{2}+\Delta_{1} \cos \left(\sqrt{\Delta_{1}+\Delta_{2}} t\right)\right], \\
\phi_{1}(t)=\cos \left(\sqrt{\Delta_{2}} t\right) . \\
\phi_{2}(t)=1 .
\end{gathered}
$$

Equation (30a) corresponds again to the harmonic behavior of the initial TACF $\phi_{0}(t)$, where the period is defined by two frequency parameters, $\Delta_{1}$ and $\Delta_{2}$.

From these two cases presented above, one can see that it is possible to find exact analytical solutions for the relaxations functions $\phi_{\nu}(t)$ at any finite dimension $\nu$.

\section{MODELS OF THE INFINITE SETS OF VARIABLES}

For the infinite-dimensional Liouville spaces, $\nu \rightarrow \infty$, the set of possible scenarios for the TACF $\phi_{0}(t)$ is vast and contains the decaying functions.

\section{A. Gaussian relaxation}

Let us now consider the case, where the frequency parameters $\Delta_{\nu}$ 's are related according to the arithmetic progression:

$$
\Delta_{1}, \quad \Delta_{2}=2 \Delta_{1}, \quad \Delta_{3}=3 \Delta_{1}, \ldots, \quad \Delta_{\nu}=\nu \Delta_{1} .
$$

Then, the continued fraction (26) takes the following form

$$
\tilde{\phi}_{0}(s)=\frac{1}{s+\frac{\Delta_{1}}{s+\frac{2 \Delta_{1}}{s+\frac{3 \Delta_{1}}{s+\ddots}}}},
$$

that corresponds in the time domain to the ordinary Gaussian function [22]

$$
\phi_{0}(t)=\mathrm{e}^{-\Delta_{1} t^{2} / 2} .
$$

The most well-known examples of the physical realization of such relaxation is the density fluctuations in the perfect gas and one-particle dynamics in liquids (at the limit of high wave numbers $k$ ) [13, 23]. Other case with this relaxation is the dynamics of the one-dimensional XY-model at $T \rightarrow \infty$ [24].

The exact correspondence between the frequency parameters [given by relations (31)] indicates on the possibility to study quantitatively the deviation from the Gaussian relaxation by means of the simple comparison of the parameters $\Delta_{\nu}$ 's:

$$
\alpha_{\nu}=\frac{\nu}{\nu+1} \frac{\Delta_{\nu+1}}{\Delta_{\nu}}-1
$$

For the Gaussian relaxation one has $\alpha_{\nu}=0$, whilst deviations from the zeroth values of $\alpha_{\nu}$ will be caused by manifestations of the non-Gaussian behavior of $\phi_{0}(t)$. 


\section{B. Damped relaxation of oscillated correlator}

Let us consider the specific case, where the frequency parameters are finite and equal to each other

$$
\Delta_{1}=\Delta_{2}=\Delta_{3}=\ldots=\Delta_{\nu}
$$

that corresponds to the continued fraction (26) of the form

$$
\tilde{\phi}_{0}(s)=\frac{1}{s+\frac{\Delta_{1}}{s+\frac{\Delta_{1}}{s+\ddots}}} .
$$

As known from the theory of continued fractions, expression (36) is the representation of the next function (over the variable $s)$ :

$$
\tilde{\phi}_{0}(s)=\frac{-s+\sqrt{s^{2}+4 \Delta_{1}}}{2 \Delta_{1}} .
$$

Applying the inverse Laplace transform operator $\hat{\mathcal{L}}^{-1}$ to equation (37), one obtains the TACF

$$
\phi_{0}(t)=\frac{1}{\sqrt{\Delta_{1}} t} J_{1}\left(2 \sqrt{\Delta_{1}} t\right),
$$

where $J_{1}$ is the Bessel function of the first order. Such relaxation appears in the processes, which characterized by the damped harmonic oscillatory behavior. For example, expression (38) is the exact form for the TACF of velocity of the Brownian particle in linear chain of the identical harmonic oscillators [1, 2, 25]. Moreover, the dynamics of the two-dimensional electron gas at the temperature $T=0$ and at the defined range of the wave number $k$ is other manifestation of relaxation with the TACF of the form (38) (see reference [26]).

Thus, the presented cases demonstrate that at the known correspondence between the frequency parameters $\mathcal{F}\left(\Delta_{1}, \Delta_{2}, \Delta_{3}, \ldots, \Delta_{\nu}, \ldots\right)$, one can exactly define the initial TACF $\phi_{0}(t)$ and to estimate the frequency features of its spectral image $\tilde{\phi}_{0}(s)$.

\section{DENSITY FLUCTUATIONS IN SIMPLE LIQUIDS}

\section{A. Frequency parameters}

Let us now consider the liquid system, where the particle interactions are characterized by the spherical symmetry and the potential contains the radial dependence only. Liquid metals and condensate noble gases are the typical examples of such systems [13, 27, 28]. Further, we take the space Fourier transform of the local density fluctuations, $A_{0}(\mathbf{k})=\rho(\mathbf{k}, t)=(1 / \sqrt{N}) \sum_{i} e^{i \mathbf{k} \cdot \mathbf{r}_{i}(t)}$, as the initial dynamical variable, the TACF of which, $\phi_{c o h}(k, t)$, is related with dynamic structure factor $S(k, \omega)$ [see equation (1)]. Then, the frequency parameters can be found according to relations (21a) and (21b)

$$
\begin{gathered}
\left(A_{0}(k), A_{0}(k)\right)=S(k), \quad\left(A_{1}(k), A_{1}(k)\right)=\frac{k_{B} T}{m} k^{2}, \\
\Delta_{1}(k)=\frac{k_{B} T}{m} \frac{k^{2}}{S(k)}=\frac{\left(v_{T} k\right)^{2}}{S(k)}, \\
\Delta_{2}(k)=3 \frac{k_{B} T}{m} k^{2}+\frac{\rho}{m} \int \nabla_{l}^{2} u(r)[1-\exp (i \mathbf{k r})] g(r) d^{3} \mathbf{r}-\Delta_{1}, \\
\Delta_{3}(k)=\frac{1}{\Delta_{2}(k)} \Xi(k)-\frac{\left[\Delta_{1}(k)+\Delta^{2}(k)\right]^{2}}{\Delta_{2}(k)},
\end{gathered}
$$


where $v_{T}$ is the average thermal velocity of particles, $\rho$ is the number density, $g(r)$ is the pair distribution function, $u(r)$ is the interparticle potential, the suffix $l$ marks the component parallel to wave vector $\mathbf{k}$. Within the assumption about pair-additivity of the interparticle potential $u(r)$, the term $\Xi(k)$ takes the following form [29]:

$$
\begin{aligned}
\Xi(k) & =15\left(\frac{k_{B} T}{m}\right)^{2} k^{4}+\frac{k_{B} T}{m} k^{2} \frac{\rho}{m} \int d \mathbf{r} g(r) \nabla_{l}^{2} u(r) \\
& +6 \rho \frac{k_{B} T}{m^{2}} k \int d^{3} \mathbf{r} g(r) \nabla_{l}^{3} u(r) \sin (\mathbf{k r}) \\
& +2 \frac{\rho}{m^{2}} \int d^{3} \mathbf{r} g(r)\left[\nabla \nabla_{l} u(r)\right]^{2}[1-\cos (\mathbf{k r})] \\
& +\frac{\rho^{2}}{m^{2}} \iint d^{3} \mathbf{r} d^{3} \mathbf{r}^{\prime} g_{3}\left(\mathbf{r}, \mathbf{r}^{\prime}\right)\left[1-\cos \left(\mathbf{k r}-\mathbf{k r}^{\prime}\right)\right]\left(\nabla \nabla_{l} u(r)\right)\left(\nabla^{\prime} \nabla_{l}^{\prime} u\left(r^{\prime}\right)\right)
\end{aligned}
$$

where $g_{3}\left(\mathbf{r}, \mathbf{r}^{\prime}\right)$ is the three-particle distribution function. The frequency parameters of a higher order will contain the more complicated integral expressions with the $n$-particles distribution functions $g_{n}(\bar{r})$, i.e. $\Delta_{\nu}(k)=$ $F\left[\Delta_{1}(k), \Delta_{2}(k), \ldots, \Delta_{\nu-1}(k) ; g(r), g_{3}(\bar{r}), \ldots, g_{\nu}(\bar{r})\right]$.

\section{B. Dynamics at high wave numbers and short time scales}

In the ranges of the wave numbers $k$ larger than $k_{m}=2 \pi / \sigma$ [where $\sigma$ is the effective particle size and $k_{m}$ is the first maximum in the static structure factor $S(k)$ ] and of the short time scales, which correspond to lengths smaller than the mean free path, the interactions between particles in simple liquids can be neglected. Taking into account that for this range of $k$ one has $S(k) \rightarrow 0$, we obtain directly from equations (39a), (39b) and (39c)

$$
\Delta_{1}(k)=\left(v_{T} k\right)^{2}, \quad \Delta_{2}(k)=2\left(v_{T} k\right)^{2}, \quad \Delta_{3}(k)=3\left(v_{T} k\right)^{2} .
$$

Comparing (41) and (31) one can see that relations (41) are the first steps of recurrence relation

$$
\Delta_{\nu+1}(k)=\frac{\nu+1}{\nu} \Delta_{\nu}(k),
$$

which obeys the Gaussian relaxation for the density autocorrelation function

$$
\phi_{c o h}(k, t)=\mathrm{e}^{-\left(v_{T} k t\right)^{2} / 2}
$$

Then, for the dynamic structure factor in the frequency domain one has the single Gaussian-like function located at the zeroth frequency. This result is completely reasonable, since it means that the short time dynamics in the range of high values of $k$ is defined by free particle movements, and the relaxation occurs over a single time scale $\tau \sim \sqrt{1 / \Delta_{1}(k)} \sim\left(v_{T} k\right)^{-1}$

\section{Microscopic dynamics at wave numbers $k \leq k_{m}$}

At the condition for wave numbers $k \leq k_{m}$, the corresponding spatial ranges of a system can be filled by few particles only. Within such the ranges, the description of the proper dynamics is relevant if it is performed in terms of two, three- and four-particle distribution functions, which are contained in the first four frequency parameters, $\Delta_{\nu}$, $\nu=1,2,3,4$.

Moreover, the treatment of experimental $I(k, \omega)$-data of inelastic X-ray scattering $\sqrt{30}]$ as well as the numerical molecular dynamics simulations results for liquid alkali metals near melting [23, 31, 32] indicate that there is the correspondence for this range of wave number:

$$
\Delta_{4}(k) \simeq \Delta_{5}(k)
$$

The extension of this equality to the frequency parameters of higher order

$$
\Delta_{\nu}=\Delta_{4}, \quad \nu>4,
$$


allows one to define the term $\tilde{\phi}_{3}(k, s)$ of the chain (25) by analogy with the model case (35)

$$
\tilde{\phi}_{3}(k, s)=\frac{-s+\sqrt{s^{2}+4 \Delta_{4}(k)}}{2 \Delta_{4}(k)} .
$$

It is necessary to note that extension (45) has a clear sense at consideration of the transition into the regime of high values of $k$. Equation (42) indicates on the equality $\Delta_{\nu}(k)=\Delta_{\nu+1}(k)$ at limit of high $\nu$-values, i.e. $\lim _{\nu \rightarrow \infty} \Delta_{\nu}(k) / \Delta_{\nu+1}(k)=1$. Thus, for the regime of $k \leq k_{m}$ the equality of frequency parameters arises at lower level of chain (23) and smaller values of $\nu$.

Then, going down over fraction (26) to the term $\tilde{\phi}_{2}(k, s)$ one obtains

$$
\tilde{\phi}_{2}(k, s)=\frac{2 \Delta_{4}(k)}{s\left[2 \Delta_{4}(k)-\Delta_{3}(k)\right]+\Delta_{3}(k) \sqrt{s^{2}+4 \Delta_{4}(k)}} .
$$

Then, the dynamic structure factor will be as the next

$$
\begin{aligned}
S(k, \omega) & =\frac{S(k)}{2 \pi} \Delta_{1}(k) \Delta_{2}(k) \Delta_{3}(k) \sqrt{4 \Delta_{4}(k)-\omega^{2}}\left\{\Delta_{1}^{2}(k) \Delta_{3}^{2}(k)\right. \\
& +\omega^{2}\left[\Delta_{1}^{2}(k) \Delta_{4}(k)-2 \Delta_{1}(k) \Delta_{3}^{2}(k)-\Delta_{1}^{2}(k) \Delta_{3}(k)+2 \Delta_{1}(k) \Delta_{2}(k) \Delta_{4}(k)-\Delta_{1}(k) \Delta_{2}(k) \Delta_{3}(k)\right. \\
& \left.\quad+\Delta_{2}^{2}(k) \Delta_{4}(k)\right] \\
& +\omega^{4}\left[\Delta_{3}^{2}(k)-2 \Delta_{1}(k) \Delta_{4}(k)+2 \Delta_{1}(k) \Delta_{3}(k)-2 \Delta_{2}(k) \Delta_{4}(k)+\Delta_{2}(k) \Delta_{3}(k)\right] \\
& \left.+\omega^{6}\left[\Delta_{4}(k)-\Delta_{3}(k)\right]\right\}^{-1} .
\end{aligned}
$$

It is necessary to note that the dynamic structure factor $S(k, \omega)$ of the form (48) reproduces the three-peak structure in the frequency domain (at the fixed $k$ ), which is observable within the experiments of inelastic neutron scattering and inelastic x-ray scattering. This spectral form is very similar to the known Rayleigh -Mandelshtam-Brillouin triplet at light scattering corresponded to the hydrodynamic limit $(t \rightarrow \infty$ and $k \rightarrow 0)$. Here, one peak of $S(k, \omega)$ is located at the zeroth frequency $(\omega=0)$, while two other peaks - the so-called doublet - are located at the finite frequencies $\left( \pm \omega_{L}\right)$.

\section{Relation with hydrodynamics}

The features of the high-frequency (inelastic) peaks of the dynamic structure factor $S(k, \omega)$ will be defined by the solution of the next equation

$$
s+\frac{\Delta_{1}(k)}{s}+\Delta_{2}(k) \tilde{\phi}_{2}(k, s)=0,
$$

which is general within the recurrence relation approach [23]. In the considered case, the function $\tilde{\phi}_{2}(k, s)$ for the presented scheme has the form (47), while equation (49) will have complex solutions $s=\Re[s(k)]+\mathrm{i} \Im[s(k)]$. Here, the imaginary part $\Im[s(k)]$ defines the high-frequency peak positions, while the real part $\Re[s(k)]$ is associated with the widths of the peaks.

To analyze equation (49) we introduce two dimensionless quantities (at the fixed $k$ ):

$$
\begin{gathered}
\xi(k)=\frac{s^{2}}{\Delta_{4}(k)}, \\
\varsigma(k)=\frac{2 \Delta_{4}(k)}{\Delta_{3}(k)}-1 .
\end{gathered}
$$

At the transition to the hydrodynamical limit the next condition should be satisfied:

$$
|\xi(k)| \ll 1,
$$


that means the consideration of the large time scales (small frequencies). Then, equation (49) takes the following form:

$$
s^{3}+\frac{2 \sqrt{\Delta_{4}(k)}}{\varsigma(k)} s^{2}+\left[\Delta_{1}(k)+\Delta_{2}(k)+\frac{\Delta_{2}(k)}{\varsigma(k)}\right] s+\frac{2 \Delta_{1}(k) \sqrt{\Delta_{4}(k)}}{\varsigma(k)}=0 .
$$

The solution of this cubic equation can be found within the convergent scheme for approximating solutions applied by Mountain (see reference [33])

$$
\begin{gathered}
s_{1,2}(k)= \pm \sqrt{\Delta_{1}(k)\left\{1+\frac{\Delta_{2}(k)[1+\varsigma(k)]}{\Delta_{1}(k) \varsigma(k)}\right\}}-\frac{\sqrt{\Delta_{4}(k)}}{\varsigma(k)}\left(1-\frac{\Delta_{1}(k) \varsigma(k)}{\Delta_{1}(k) \varsigma(k)+\Delta_{2}(k) \varsigma(k)+\Delta_{2}(k)}\right), \\
s_{3}(k)=-\frac{2 \Delta_{4}(k)}{\varsigma(k)} \frac{\Delta_{1}(k) \varsigma(k)}{\Delta_{1}(k) \varsigma(k)+\Delta_{2}(k) \varsigma(k)+\Delta_{2}(k)} .
\end{gathered}
$$

These approximated solutions corresponds to the results of the hydrodynamical Landau-Placzek theory with the following parameters: the adiabatic sound velocity $c_{s}$ is

$$
\left(c_{s} k\right)^{2}=\lim _{k \rightarrow 0}\left(v_{T} k\right)^{2}=\lim _{k \rightarrow 0} \Delta_{1}(k),
$$

the sound damping parameter is

$$
\Gamma=\frac{\gamma-1}{\gamma} \frac{\sqrt{\Delta_{4}(k)}}{\varsigma(k)}
$$

where the ratio of the specific heat at constant pressure to the specific heat at constant volume $\gamma=c_{p} / c_{v}$ is

$$
\gamma=1+\frac{\Delta_{2}(k)+\Delta_{2}(k) \varsigma(k)}{\Delta_{1}(k) \varsigma(k)} .
$$

Thus, the expression for the dynamic structure factor $S(k, \omega)$ of the form (48) satisfies completely the transition into hydrodynamic regime; and the parameters of hydrodynamic theory are expressed through the frequency parameters $\Delta_{1}(k), \Delta_{2}(k), \Delta_{3}(k)$ and $\Delta_{4}(k)$.

\section{CONCLUSIONS}

In this paper, we have presented the approach, by means of which the relaxation processes in many particle systems can be studied. This approach reformulates the equation of motion for the considered dynamical variable in terms of the recurrence relations, where the set of frequency parameters appear. In fact, the recurrence relations approach states that if the frequency parameters or the correspondence between of them are known, then the solution for the TACF can be found. Moreover, it imposes the general routine to define these frequency parameters; however, the specific analytical expressions depend on a concrete problem studied [26, 34 36].

Here, we have shown some simple model cases with the exact solutions, which are realized, nevertheless, in the physical problems. As the other nontrivial case, we have considered the problem of the description of local density fluctuations in simple liquids. As it was shown, the solution for the spectral image of the local density fluctuation TACF can be obtained within the recurrence relations, and this solution is correctly consistent with the known asymptotic scenarios.

The important advantage of the approach is the absence of the abstract parameters at the construction of theoretical description. All the quantities are expressed only through the frequency parameters, which contain the details of interparticle interactions and static structure correlations. Therefore, such the approach, in our opinion, is the perfect one within of which it is possible to construct the explanation how the many particle system structure defines the system dynamics. 


\section{ACKNOWLEDGEMENTS}

We would like to thank M. Howard Lee (University of Georgia, USA) for very useful discussions.

[1] Zwanzig, R. (2001) Nonequilibrium Statistical Mechanics, Oxford University Press: Oxfrod.

[2] Mokshin, A.V., Yulmetyev, R.M. and Hänggi, P. (2005), Simple Measure of Memory for Dynamical Processes Described by a Generalized Langevin Equation, Physical Review Letters, 95, 200601(1)-200601(4).

[3] Uchaikin, V.V. (2003), Self-similar anomalous diffusion and Levy-stable laws, Physics-Uspekhi, 46, 821-849.

[4] Risken, H. (1989), The Fokker-Planck equation. Methods of solution and applications, 2nd ed., Springer-Verlag: Berlin.

[5] Gammaitoni, L., Hänggi, P., Jung, P., Marchesoni, F. (1998) Stochastic resonance, Review of Modern Physics, 70, $223-287$.

[6] Berthier, L., et al. (2011) Dynamical heterogeneities in glasses, colloids, and granular media, Oxford University Press: Oxford.

[7] Berne, B.J. and Harp, G.D. (1970), On the Calculation of Time Correlation Functions, Advances in Chemical Physics XVII, 63, 63-227.

[8] Kubo, R. (1957), Statistical-Mechanical Theory of Irreversible Processes. I. General Theory and Simple Applications to Magnetic and Conduction Problems, Journal of the Physical Society of Japan, 12, 570-586.

[9] Zausch, J. et al. (2008), From Equilibrium to Steady State: The Transient Dynamics of Colloidal Liquids under Shear, J. Phys.: Condens. Matter, 20, 404210.

[10] Rahman, R. (1964), Correlations in the Motion of Atoms in Liquid Argon, Physical Review A, 136, 405-411.

[11] Lee, M.H. (2007), Why Irreversibility Is Not a Sufficient Condition for Ergodicity, Physical Review Letters, 98, 190601.

[12] Callen, H.B., Welton, T.R. (1951), Irreversibility and Generalized Noise, Physical Review, 83, 34-40.

[13] Hansen, J. P., McDonald, I. R. (2006), Theory of Simple Liquids, Academic Press: New York.

[14] Mokshin, A.V., Chvanova, A.V., and Khusnutdinoff, R.M. (2012), Mode-coupling approximation in a fractional-power generalization: Particle dynamics in supercooled liquids and glasses, Theoretical and Mathematical Physics, 171, 541-552.

[15] Götze, W. (2009), Complex Dynamics of Glass-Forming Liquids - A mode-coupling theory, Oxfrod University Press Inc.: New York.

[16] Balucani, U., Lee, M.H., Tognetti, V. (2003), Dynamical Correlations, Physics Report, 373, 409-492.

[17] Lee, M.H. (1983), Derivation of the generalized Langevin equation by a method of recurrence relations, Journal of Mathematical Physics, 24, 2512-2514.

[18] Feldman, Yu., Puzenko, A. and Ryabov, Ya. (2006), Dielectric Relaxation Phenomema in Complex Materials in Fractals, Diffusion, and Relaxation in Disordered Complex Systems: A Special Volume of Advances in Chemical Physics. vol. 133, Part A, edited by Coffey, W.T. and Kalmykov, Yu.P.

[19] Lee, M.H. (2000), Generalized Langevin equation and recurrence relations, Physical Review E, 62, 1769-1772.

[20] Lee, M.H. (2000), Heisenberg, Langevin, and current equations via the recurrence relations approach, Physical Review E, 61, 3571-3578.

[21] Jr. Florencio, J. and Lee, M.H. (1985), Exact time evolution of a classical harmonic-oscillator chain, Physical Review A, 31, 3231-3236.

[22] Abramowitz, M. and Stegun, I.S. (1972), Handbook of Mathematical Functions, Dover: New York.

[23] Mokshin, A.V., Yulmetyev, R.M., Khusnutdinoff, R.M., and Hänggi P. (2006) Analysis of the dynamics of liquid aluminium: Recurrent relation approach, Journal of Physics Condensed Matter, 19(4), 046209(1)-046209(16).

[24] Jr. Florencio, J. and Lee, M.H. (1987), Relaxation functions, memory functions, and random forces in the one-dimensional spin 1/2-XY and transverse Ising models, Physical Review B, 35, 1835-1840.

[25] Rubin, R.J. (1963), Momentum Autocorrelation Functions and Energy Transport in Harmonic Crystals Containing Isotopic Defects, Physical Review, 131, 964-989.

[26] Lee, M.H., Hong, J. and Jr. Florencio, J. (1987), Method of Recurrence Relations and Applications to Many-Body Systems, Physica Scripta, T19, 498-504.

[27] Yulmetyev, R.M., Mokshin, A.V., Hänggi, P. and Shurygin, V.Yu. (2002), Dynamic Structure Factor in Liquid Cesium on the Basis of Time-Scale Inveriance of Relaxation Processes, JETP Letters, 76, 147-150.

[28] Yulmetyev, R.M., Mokshin, A.V., Hänggi, P. and Shurygin, V.Yu. (2001), Time-scale invariance of relaxation processes of density fluctuation in slow neutron scattering in liquid cesium, Physical Review E, 64, 057101(1)-057101(4).

[29] Bansal, R. and Pathak, K.N. (1974), Sum rules and atomic correlations in classical liquids, Physical Review A, 9, $2773-2782$.

[30] Yulmetyev, R.M., Mokshin, A.V., Scopigno, T. and Hänggi, P. (2003), New evidence for the idea of time-scale invariance of relaxation processes in simple liquids: the case of molten sodium, Journal of Physics: Condensed Matter, 15, 2235-2257.

[31] Khusnutdinoff, R.M., Mokshin, A.V. and Yulmetyev, R.M. (2009), Molecular Dynamics of Liquid Lead near its Melting Point, Journal of Experimental and Theoretical Physics, 108, 417-427.

[32] Mokshin, A.V., Yulmetyev, R.M. Khusnutdinoff, R.M. and P. Hänggi (2006), Collective Dynamics in Liquid Aluminum near the Melting Temperature: Theory and Computer Simulation, Journal of Experimental and Theoretical Physics, 103, 841-849.

[33] Mountain, R.D. (1966), Spectral Distribution of Scattered Light in a Simple Fluid, Review of Modern Physics, 38, 205-214. [34] Wierling, A. (2012), Dynamic structure factor of linear harmonic chain - a recurrence relation approach, The European 
Physical Journal B, 85, 1-9.

[35] Wierling, A., Sawada, I. (2010), Wave-number dependent current correlation for a harmonic oscillator, Physical Review E, 82, 051107(1)-051107(11).

[36] Wierling, A., Sawada, I. (2012), Dynamic Structure Factor for a Harmonic Oscillator and the Harmonic Oscillator Chain, Contributions to Plasma Physics, 52, 49-52. 